Иркутский национальный исследовательский технический университет, 2. Иркутск, Российккая Федераиия

Е. Г. Тарханова

Байкальский государственный университет,

г. Иркутск, Российкая Федераиия

\title{
ИНФОРМАЦИОННЫЕ ТЕХНОЛОГИИ В СФЕРЕ ОБСЛУЯКИВАНИЯ КАК НАПРАВЛЕНИЕ ИННОВАЦИОННОЙ ДЕЯТЕЛЬНОСТИ (НА ПРИМЕРЕ ИНДУСТРИИ ГОСТЕПРИИМСТВА)
}

\begin{abstract}
АНнотАция. В современных условиях экономической реальности информационные технологии являются фактором обеспечения әффективного производства и конкурентоспособности предприятия, а также индикатором его инновационной активности. Анализ современной экономической научной литературы свидетельствует о наличии ряда дискуссионных вопросов касательно осуществления инновационной деятельности на основе применения информационных технологий в сфере обслуживания и, в частности, в таком межотраслевом секторе сферы услуг, как индустрия гостеприимства. В статье проанализирована тенденция развития сферы услуг, затронуто понятие сервисных инноваций, отличных от производственных. Сделан вывод о том, что характер инноваций в сфере обслуживания зависит от специфики деятельности предприятия. В качестве одного из важнейших факторов эффективности инноваций в индустрии гостеприимства отмечена клиентоориентированность персонала. Выделены и рассмотрены две группы информационных технологий, применяемых на предприятиях индустрии гостеприимства, отмечены препятствия к внедрению новшеств и рассмотрен так называемый «парадокс информационных технологий» в сфере обслуживания.
\end{abstract}

кЛЮЧЕВЫЕ СЛОВА. Информационные технологии; инновации; индустрия гостеприимства; сфера услуг.

ИНФОРМАЦИЯ О СТАТЬЕ. Дата поступления 14 марта 2016 г.; дата принятия к печати 30 марта 2016 г.; дата онлайн-размещения 31 мая 2016 г.

Ye. O. Pokhomchikova Irkutsk National Research Technical University, Irkutsk, Russian Federation

Ye. G. Tarkhanova Baikal State University, Irkutsk, Russian Federation

\section{INFORMATION TECHNOLOGIES IN SERVICES SECTOR AS A DIRECTION LINE OF INNOVATION ACTIVITY (IN THE CONTEXT OF HOSPITALITY INDUSTRY)}

\begin{abstract}
Under current conditions of economic reality, information technologies are the factor of providing effective production and competitiveness of a company, as well as an indicator of its innovative activity. An analysis of present-day economic scientific literature testifies presence of a number of debatable issues in regard of implementing innovative activity on the basis of using information technologies in the services sector and, in particular, in such an interindustrial sector of services as the hospitality industry. The article analyses the development tendencies for the services sector, considers the concept of services innovations that are different from the industrial ones. It makes a conclusion that the character of innovations in the services sector depends on the specificity of a company's activity. As one of the most important factors of innovation efficiency in the hospitality industry, it notes the client-orientedness of the personnel.
\end{abstract}

\section{Baikal Research Journal}


It identifies and considers two groups of information technologies used in the hospitality industry companies, ear-marks the obstacles to introducing novelties and considers the so-called «paradox of information technologies» in the services sector.

KEYWORDS. Information technologies; innovations; hospitality industry; services sector. ARTICLE INFO. Received March 14, 2016; accepted March 30, 2016; available online May 31, 2016.

Информационные технологии (ИТ) являются неотъемлемым атрибутом развития как отдельных отраслей, так и экономики в целом. Этот факт еще раз свидетельствует о том, что устойчивый рост возможен, в том числе при тесном взаимодействии технико-технологической и экономической составляющих. Результатом такого взаимодействия становится разработка прикладных информационных программ и технологий, позволяющих значительно повысить эффективность деятельности предприятий.

Объектом исследования в статье являются ИТ в сфере обслуживания, а именно в индустрии гостеприимства. Выбор сервисной сферы деятельности в качестве субъекта применения ИТ основан на следующих положениях:

1. Обозначившаяся со второй половины XX столетия тенденция динамического роста сферы услуг. Так, по оценкам Всемирного банка, на долю сервисного сектора приходится примерно 70 \% мирового валового внутреннего продукта (ВВП) ${ }^{1}$.

2. Тренд трансформации структуры российского ВВП в сторону увеличения доли сферы услуг. С 1990 г. доля услуг в общем объеме ВВП России возросла с 34,9 до 60,4 \% в 2003 г. $^{2}$ (индекс роста - 1,7). Несмотря на то, что данная доля с 2003 г. остается относительно стабильной (в пределах 60 \%), структура сферы услуг изменяется. Это связано как с появлением новых услуг и изменением старых, так и с укреплением позиций существующих [1, с. 87]. В конце 90-х гг. ХХ в. в российский лексикон устойчиво вошло словосочетание «индустрия гостеприимства». Многие вопросы, связанные с аспектами функционирования данной индустрии, в том числе ее структуры, до сих пор остаются дискуссионными, что подтверждает актуальность проведения дальнейших исследований. Как правило, в составе индустрии гостеприимства выделяют предприятия сферы гостиничного сервиса, общественного питания, туризма и развлечений [2, с. 15]. Полагаясь на указанную структуру, необходимо отметить перспективность развития данных предприятий, особенно в сложившихся нелегких условиях политико-экономической ситуации в России, высокого курса иностранных валют, способствующих переключению населения на потребление услуг внутри страны.

3. Ощущающаяся нехватка исследований в области сервисного менеджмента, в качестве одной из причин которой можно выделить недооцененность роли сферы услуг в развитии экономики [3, с. 37].

4. Отнесение разработки и применения ИТ к инновационному направлению деятельности, являющейся актуальной для развития предприятий сферы обслуживания, в особенности индустрии гостеприимства, исследование вопросов инновационной деятельности которой до сих пор находится на стадии формирования $[4$, с. $18 ; 5$, с. 3$]$.

Общеизвестным является тот факт, что инновации выступают одним им из мощнейших драйверов развития экономики, а также обеспечения конкурентоспособности. Несмотря на то, что данная фраза является широко употребляемой как в научной, так и публицистической литературе и иных средствах массовой информации, думается, она требует конкретизации, а именно уточнения: что лежит в основе инно-

${ }^{1}$ The World Bank. URL : http://www.worldbank.org/.

${ }^{2}$ Структура ВВП // Федеральной службы государственной статистики. URL : http://www.gks.ru/ wps/wcm/connect/rosstat_main/rosstat/ru/statistics/accounts/\#.

\section{Baikal Research Journal}


ваций на каждой стадии развития экономики. Если для экономики индустриального типа основным фактором производства были ресурсы и новые источники энергии, то для экономики нового типа (так называемой новой экономики, экономики знаний) в качестве этого фактора выступают новые знания и основанные на них технологии. Таким образом, технологические инновации в настоящее время являются фактором, определяющим производительность в различных отраслях экономики [6, с. 3-4].

Согласно большинству из представленных в литературе классификаций инноваций, ИТ относятся к виду «технико-технологические инновации». Однако, думается, в качестве основополагающего признака классификации должен выступать тип производства: промышленность (сфера производства) и сфера услуг. Соответственно, в самом укрупненном виде инновации можно разделить на производственные и сервисные. Вопрос разграничения данных типов инноваций до сих пор остается дискуссионным. Условно исследователей, занимающихся изучением инноваций, можно разделить на два лагеря. Первые считают, что между производственными и сервисными инновациями не существует принципиальной разницы, вторые, наоборот, полагают, что сама специфика сферы услуг накладывает отпечаток на применяемые в ней инновации, как следствие сервисные инновации имеют отличия от производственных и требуют разработки специальных методов оценки и инструментов политики $[7$, с. 4]. Мы разделяем точку зрения представителей второго подхода и считаем, что вид деятельности предприятий сферы обслуживания также налагает определенные особенности на реализуемые инновации. Так, применительно к индустрии гостеприимства, ключевой характеристикой инноваций является клиентоориентированность, тогда как аспект новизны внедряемых новшеств относится к факторам более низкого порядка [8].

До 90-х гг. XX в. ученое сообщество уделяло мало внимания изучению проблемы сервисных инноваций ${ }^{3}$ В то время инновации воспринимались исключительно как технологические новшества, создающиеся и применяющиеся в обрабатывающей промышленности [9-12]. «Так, по данным pecypca Publish or Perish, в 1975-1989 гг. словосочетание "сервисные инновации" встречалось в заголовках всего восьми статей. В период с 1990 по 1999 гг. таких работ было 24, а с 2000 по 2009 гг. - около $500{ }^{4}$. Несмотря на возможные неточности в выборке, данная публикационная тенденция является довольно показательной [7, с. 6]. Таким образом, возросший в последнее время интерес к сервисным инновациям является своего рода приведением акцентов инновационной политики в сферах производства и услуг в относительное равновесие.

Что касается технико-технологического типа сервисных инноваций, а именно ИТ в сфере обслуживания, можно отметить, что уже к 80-х гг. XX столетия сферу услуг начали относить к одному из самых активных пользователей информационных технологий $[7$, с. 5]. С течением времени в ряде ее отраслей появлялись абсолютно новые сервисы, основанные на применении ИТ: торговые, банковские, гостиничные, ресторанные, туристские, издательские, в том числе коммуникационные (CRM). Таким образом, роль сферы услуг начала трансформироваться от пассивного пользователя к генератору развития отрасли ИТ. Появление Интернета лишь усилило эту тенденцию.

До недавнего момента так называемый комплексный подход к информационной инфраструктуре предприятий индустрии гостеприимства был, скорее, исключением из правил, являясь прерогативой фешенебельных гостиниц и ресторанов класса

${ }^{3}$ Понятие «сервисные инновации» пришло из зарубежной литературы, в которой в качестве сервисных обозначаются инновации в сфере услуг. В отечественной литературе данный термин не нашел распространения, что, по мнению авторов, является существенным упущением. Полагаем, что для идентификации инноваций, сопряженных с услуговой дея-тельностью, следует использовать термин "сервисные инновации».

${ }^{4}$ Publish or Perish. Version 3.0.3883 (18 August). URL : http://www.harzing.com/pop.htm.

\section{Baikal Research Journal}

электронный научный журнал Байкальского государственного университета 
«люкс». Однако постоянно возрастающие требования клиентов диктуют необходимость внесения серьезных изменений в концепцию функционирования указанных предприятий. В последние годы инвесторы проектов сферы гостеприимства все чаще начали обращать внимание на фактор использования прогрессивных ИТ в деятельности предприятия. «При реконструкции существующих гостиниц в отдельную категорию работ выделяют не только дизайн помещений и замену инженерных систем, но и построение современной информационной инфраструктуры, а при строительстве новых объектов соответствующий раздел утверждается на стадии проектирования» ${ }^{\text {. }}$

На сегодняшний день существует множество направлений применения ИТ в индустрии гостеприимства. Условно их можно разделить на две группы:

1. ИТ, поддерживающие и оптимизирующие производственные процессы предприятия:

- система управления электроэнергией и освещением, позволяющая, в среднем, сократить потребление ресурсов примерно на 30 \% [13];

- система управления внутренними службами отеля (Back-office), позволяющая повысить эффективность работы персонала, тем самым улучшить качество обслуживания постояльцев. Суть системы состоит в том, что карманный персональный компьютер каждого сотрудника настроен на беспроводную систему передачи информации с центрального компьютера: о количестве товаров на складе, о номерах, требующих уборки, ремонте, а также об особых пожеланиях гостей [13];

- технология ЛЭД-оповещения (экран со светодиодной подсветкой), позволяющая своевременно уведомлять рабочих о выполненных задачах: готовности блюд, тех или иных функций;

- компьютерные системы централизованного управления, позволяющие автоматизировать основные этапы работы гостиницы: резервирование номеров, регистрация, размещение и выписка гостей, управление номерным фондом, ведение бухгалтерского учета и т. д. ${ }^{6}$;

- инновационный веб-мониторинг - система обнаружения и регистрации любого несоблюдения санитарных норм и правил безопасности, которая осуществляет запись места приготовления пищи, фиксирует нарушения в работе сотрудника ${ }^{7}$.

2. ИТ, направленные на удовлетворение постоянно возрастающих запросов клиентов и позволяющие выстраивать долгосрочные отношения с потребителями услуг:

- системы интерактивного телевидения;

- выносная панель аудио-, видеоразъемов (Connectivity Panel) - устройство, позволяющее гостю подключать свое оборудование [13];

- интеллектуальная система «умный дом»;

- интеллектуальные замки, не требующие ключа и идентифицирующие гостя по радужной оболочке глаза или отпечаткам пальцев;

- CRM-система - система правления взаимоотношениями с клиентами (Customer Relationship Management System), основанная на применении новых управленческих и информационных технологий, с помощью которых предприятие аккумулирует информацию о клиентах для выстраивания взаимовыгодных и долгосрочных отношений с ними [14, с. 89];

- глобальные системы бронирования;

- вендинговые автоматы - устройства по продаже товаров без помощи продавца;

${ }^{5}$ Современная инженерная инфраструктура гостиниц : метод. пособие. URL : http://golovin.narod. $\mathrm{ru} /$ Verstka.pdf.

${ }^{6}$ Пять инновационных технологий, изменивших ресторанный бизнес // Предприниматель : офиц. сайт бизнес-портала. URL : http://business-opening.ru/franchises $/ \%$ D1 $\% 81 \% \mathrm{D} 1 \% 2 \% \mathrm{D} 0 \% \mathrm{~B} 0 \% \mathrm{D} 1 \%$ $82 \% \mathrm{D} 1 \% 8 \mathrm{C} \% \mathrm{D0} \% \mathrm{~B} 8 / 555275-2 /$.

${ }^{7}$ URL : http://business-opening.ru/franchises/\% D1 \% 81\% D1 \% 82\% D0\% B0 \% D1 \% 82\% D1 \% 8C $\%$ D $0 \% \mathrm{~B} 8 / 555275-2 /$.

\section{Baikal Research Journal}


- система приема цифровых заказов, основанная на вмонтированной в стол сенсорной панели;

- доставка блюда до потребителя напрямую из кухни через систему рельс.

Безусловно, направлений внедрения информационных технологий в индустрии гостеприимства гораздо больше, однако даже по представленным примерам можно судить об их многообразии. Следует также отметить, что большинство из перечисленных инноваций обладают синергетическим эффектом: они позволяют оптимизировать работу предприятия и повысить удовлетворенность клиентов услугами.

Необходимо отметить и имеющиеся препятствия к внедрению новшеств на предприятиях индустрии гостеприимства:

1. Стоимость инновационной разработки. Большинство разработок представлено зарубежными производителями, как следствие, покупка импортных технологий является довольно затратной, особенно в сложившихся условиях социально-политической нестабильности.

2. Необходимость адаптации предприятия к новшеству .

3. Сложность обоснования необходимости осуществления инвестиционных затрат, а также существующие риски.

4. Необходимость обучения персонала.

Помимо всего прочего, многие эксперты отмечают, что внедрение инновационных разработок в индустрии гостеприимства не всегда является обоснованным [4, с. 17]. Объяснением этому является так называемый «парадокс информационных технологий», исследованный американским экономистом Р. Солоу. Ученый выявил, что эффект от внедрения новой компьютерной техники не пропорционален объему осуществленных на закупку этой техники инвестиций [15]. По данным Р. Солоу, в США в «80-е годы XX столетия в компьютерную технику был инвестирован 1 трлн дол., при этом на сферу услуг приходилось 85 \% этой суммы, но производительность труда в ней увеличилась менее чем на 1 \% в годовом исчислении» ${ }^{8}$. Комитет Национальной академии наук США выдвинул несколько причин объяснения этого парадокса:

1. Затратное и неэффективное использование информационных технологий. Эту причину можно рассматривать с нескольких сторон. Во-первых, часто технологии используются для автоматизации неэффективных систем и процессов. Во-вторых, эффективность использования технологий во многом зависит от так называемого человеческого фактора, а, следовательно, и от компетентности сотрудников.

2. Влияние иных факторов на производительность.

3. Устаревшие методы оценки производительности.

4. Долгосрочный характер инвестиций.

5. Уровень агрегирования. При исследовании парадокса Р. Солоу рассматривал влияние технологических расходов на производительность на уровне предприятия, а не на макроуровне.

В качестве заключения хотелось бы привести высказывание директора информационного отдела корпорации Х. П. Страссмана: «Отсутствие корреляции затрат на внедрение информационных технологий с финансовыми результатами привело меня к выводу, что причина этого заложена не в компьютерах, а в людях, которые с ними работают... Проблема заключается не в свойственных технологиям возможностях, которые не перестают удивлять, а в управленческой неспособности персонала использовать их эффективно» ${ }^{9}$. По нашему мнению, ИТ дают возможность систематизировать деятельность предприятия и вывести ее на новый более высокий уровень, однако, возможно, это лишь при условии качественной, эффективной и клиентоориентированной работы персонала.

\footnotetext{
${ }^{8}$ Информационные технологии в сфере услуг. URL : http://studopedia.ru/9_7679_informatsionnietehnologii-v-sfere-uslug.html.

${ }^{9}$ URL : http://studopedia.ru/9_7679_informatsionnie-tehnologii-v-sfere-uslug.html.
}

\section{Baikal Research Journal}




\section{Список использованной литературы}

1. Институциональные изменения в сфере социально значимых услуг / Т. Д. Бурменко, В. Ю. Баганов, О. Н. Баева [и др.] ; отв. ред. Т. А. Кондрацкая.. - Иркутск : Изд-во БГУЭП, 2013. $-268 \mathrm{c}$.

2. Елканова Д. И. Основы индустрии гостеприимства : учеб. пособие / Д. И. Елканова, Д. А. Осипов, В. В. Романов. - М. : Дашков и К을 $2009 .-248$ с.

3. Бурменко Т. Д. Сфера услуг: экономика : учеб. пособие / Т. Д. Бурменко, Н. Н. Даниленко, Т. А. Туренко. - М. : Кнорус, 2007. - 322 с.

4. Немковский Б. Л. Инновации: способность предложить новое качество / Б. Л. Немковский // Отель. - 2010. - № 7. - С. 17-22.

5. Похомчикова Е. О. Индустрия гостеприимства: эволюционный подход : автореф. дис. ... канд. экон. наук : 08.00.05 / Е. О. Похомчикова. - Иркутск, 2015. - 24 с.

6. Рожкова Е. В. Формирование инновационно-ориентированного подхода к развитию сферы услуг: теория и прикладные аспекты в здравоохранении : автореф. дис. ... д-ра экон. наук : 08.00.05 / Е. В. Рожкова. - Ульяновск, 2012. - 46 с.

7. Майлс Й. Сервисные инновации в XXI веке / Й. Майлс // Форсайт. — 2011. — № 2. C. 4-15.

8. Анфилатова Ю. Ю. Информационные технологии в сфере гостеприимства / Ю. Ю. Анфилатова // Управление инновациями: теория, методология, практика : материалы VII Междунар. науч.-практ. конф. / под общ. ред. С. С. Чернова. - Новосибирск: Изд-во Центра развития науч. сотрудничества, 2014. - C. 80-89. — DOI : 978-54-00068-051-3.

9. Barras R. Towards a Theory of Innovation in Services / R. Barras // Research Policy. 1986. - № 15. - P. 161-173.

10. Chang S. Promoting innovation in hospitality companies through human resource management practices / S. Changa, Y. Gongb, C. Shumb // International Journal of Hospitality Management. - 2011. - № 30 (4). - P. 812-818.

11. Nicolaua J. L. The effect of innovation on hotel market value / J. L. Nicolau, M. J. SantaMaria // International Journal of Hospitality Management. — 2013. — Iss. 32. — P. 71-79.

12. Radu E. Innovation in the hospitality industry [Electronic resource] / E. Radu. - Mode of access : http://www.academia.edu.

13. Черноусова Н. Ю. Основные направления внедрения инновационных технологий в сфере гостеприимства [Электронный ресурс] / Н. Ю. Черноусова / Сервис в России и за рубежом. - 2012. - № 2. - Режим доступа : http://tourlib.net/statti_tourism/chernousova.htm.

14. Драгунова И. В. Информационные технологии в сфере услуг / И. В. Драгунова // Вестник Волжского университета им. В. Н. Татищева. - 2009. - № 16. - С. 89-95.

15. Платонов В. В. «Парадокс Солоу» двадцать лет спустя, или об исследовании влияния инноваций в информационных технологиях на рост производительности / В. В. Платонов // Финансы и бизнес. - 2007. - № 3. - С. 28-38.

\section{References}

1. Burmenko T. D., Baganov V. Yu., Bayeva O. N. et al.; Kondratskaya T. A. (ed.). Institutsional'nye izmeneniya $v$ sfere sotsial'no znachimykh uslug [Institutional changes in the sphere of socially significant services]. Irkutsk, Baikal State University of Economics and Law Publ., 2013. $268 \mathrm{p}$.

2. Elkanova D. I., Osipov D. A., Romanov V. V. Osnovy industrii gostepriimstva [Basics of hospitality industry]. Moscow, Dashkov i K ${ }^{\circ}$ Publ., 2009. 248 p.

3. Burmenko T. D., Danilenko N. N., Turenko T. A. Sfera uslug: ekonomika [Services sector: economy]. Moscow, Knorus Publ., 2007. 322 p.

4. Nemkovskii B. L. Innovations: ability to offer a new quality. Otel' $=$ Hotels, 2010, no. 7, pp. 17-22. (In Russian).

5. Pokhomchikova E. O. Industriya gostepriimstva: evolyutsionnyi podkhod. Avtoref. Kand. Diss. [Hospitality industry: evolutionary approach. Cand. Diss. Thesis]. Irkutsk, 2015. 24 p.

6. Rozhkova E. V. Formirovanie innovatsionno-orientirovannogo podkhoda $k$ razvitiyu sfery uslug: teoriya i prikladnye aspekty $v$ zdravookhranenii. Avtoref. Kand. Diss. [Building innovation-oriented approach to developing the services sector: theory and applied aspects in healthcare. Cand. Diss. Thesis]. Ul'yanovsk, 2012. 46 p.

\section{Baikal Research Journal}


7. Mails I. Service innovations in XXI century. Forsait $=$ Foresight and STI Governance, 2011, no. 2, pp. 4-15. (In Russian).

8. Anfilatova Yu. Yu. Information technologies in the hospitality sector. In Chernov S. S. (ed.). Upravlenie innovatsiyami: teoriya, metodologiya, praktika. Materialy VII Mezhdunarodnoi nauchno-prakticheskoi konferentsii [Innovation management: theory, methodology, practice. Materials of the 7th International Research Conference]. Novosibirsk, Development Center for Scientific Publ., 2014, pp. 80-89. DOI: 978-54-00068-051-3. (In Russian).

9. Barras R. Towards a Theory of Innovation in Services. Research Policy, 1986, no. 15, pp. 161-173.

10. Chang S., Gongb Y., Shumb C. Promoting innovation in hospitality companies through human resource management practices. International Journal of Hospitality Management, 2011 , no. $30(4)$, pp. 812-818.

11. Nicolaua J. L., Santa-Maria M. J. The effect of innovation on hotel market value. International Journal of Hospitality Management, 2013, iss. 32, pp. 71-79.

12. Radu E. Innovation in the hospitality industry. Available at: http://www.academia.edu.

13. Chernousova N. Yu. Main directions of introducing information technologies in the hospitality industry. Servis $v$ Rossii $i$ za rubezhom $=$ Services in Russia and Abroad, 2012, no. 2. Available at: http://tourlib.net/statti_tourism/chernousova.htm. (In Russian).

14. Dragunova I. V. Information technologies in the services sector. Vestnik Volzhskogo universiteta im. V. N. Tatishcheva = Bulletin of Volzhsky University after V.N. Tatishchev, 2009, no. 16, pp. 89-95. (In Russian).

15. Platonov V. V. «Paradox Solow» twenty years later, or about investigating the influence of innovations in information technologies on production growth. Finansy $i$ biznes = Finance and Business, 2007, no. 3, pp. 28-38. (In Russian).

\section{Информация об авторах}

Похолчикова Елена Олеговна - кандидат экономических наук, доцент, кафедра технологии продуктов питания и химии, Иркутский национальный исследовательский технический университет, 664074, г. Иркутск, ул. Лермонтова, 83, e-mail: Elena.isea@mail.ru.

Тарханова Елена Геннадьевна - кандидат экономических наук, доцент, кафедра менеджмента, маркетинга и сервиса, Байкальский государственный университет, 664004, г. Иркутск, ул. Ленина, 11, e-mail: Lenatarhanova@mail.ru.

\section{Authors}

Yelena O. Pokhomchikova - PhD in Economics, Associate Professor, Chair of Chemistry and Food Technology, Irkutsk National Research Technical University, 83 Lermontov St., 664074, Irkutsk, Russian Federation; e-mail: Elena.isea@mail.ru.

Yelena G. Tarkhanova - PhD in Economics, Associate Professor, Chair of Management, Marketing and Services, Baikal State University, 11 Lenin St., 664003, Irkutsk, Russian Federation; e-mail: Lenatarhanova@mail.ru.

\section{Библиографическое описание статьи}

Похомчикова Е. О. Информационные технологии в сфере обслуживания как направление инновационной деятельности (на примере индустрии гостеприимства) / Е. О. Похомчикова, Е. Г. Тарханова // Baikal Research Journal. - 2016. - T. 7, № 3. - DOI : 10.17150/2411$\underline{6262.2016 .7(3) .14}$.

\section{Reference to article}

Pokhomchikova Ye. O., Tarkhanova Ye. G. Information technologies in services sector as a direction line of innovation activity (in the context of hospitality industry). Baikal Research Journal, 2016, vol. 7, no. 3. DOI : 10.17150/2411-6262.2016.7(3).14. (In Russian).

\section{Baikal Research Journal}

